\title{
CONTRIBUCIÓN AL ESTUDIO FITOQUÍMICO DE LOS TUBÉRCULOS DE Ullucus tuberosus CALDAS
}

\author{
Ingrit Elida Collantes Díaz ${ }^{* 1}$, Elena Alicia Cóndor Cuyubamba ${ }^{2}$
}

\begin{abstract}
RESUMEN
Este trabajo describe el análisis fitoquímico del zumo liofilizado de los tubérculos de Ullucus tuberosus, nativos de la región andina, los cuales son usados en la gastronomía peruana. A partir de la extracción con solventes orgánicos y separación por transesterificación de los ácidos grasos y métodos cromatográficos, fueron identificados por primera vez la presencia del éster metílico del ácido palmítico y los esteroides estigmasterol y espinasterol en la especie en estudio, estos compuestos fueron identificados por análisis de sus espectros de resonancia magnética nuclear de hidrógeno-1 y de carbono-13.
\end{abstract}

Palabras clave: Ullucus tuberosus, tubérculos, estigmasterol, espinasterol.

\section{CONTRIBUTION TO THE PHYTOCHEMICAL STUDY OF TUBERS FROM Ullucus tuberosus CALDAS}

\begin{abstract}
This paper describes the phytochemical analysis of the lyophilized juice of the tubers from Ullucus tuberosus, native of the Andean region, which are used in Peruvian gastronomy. From organic solvents extraction and separation by transesterification of fatty acids and chromatography methods, the presence of palmitic acid methyl ester and stigmasterol and spinasterol steroids in the species under study were first identified, these compounds were identified by analysis of its nuclear magnetic resonance spectra hydrogen-1 and carbon-13.
\end{abstract}

Key words: Ullucus tuberosus, tuber, stigmasterol, spinasterol

\footnotetext{
a Facultad de Ingeniería Química y Textil, Universidad Nacional de Ingeniería, Av. Túpac Amaru No 210, Lima, Perú, ingrit_uni@hotmail.com

b Facultad de Ciencias, Universidad Nacional de Ingeniería.
} 


\section{INTRODUCCIÓN}

El Perú es un país que presenta una amplia flora, es muy rico en leguminosas (todas las menestras de consumo humano y otras), gramíneas (la amplia variedad de maíz) y de tubérculos, entre los cuales tenemos la gran variedad de papa, oca, ollucos, etc. Todos ellos consumidos por los seres humanos y son esenciales en su dieta diaria ${ }^{1}$.

El Ullucus tuberosus Caldas es una planta herbácea que pertenece al género Ullucus de la familia de las Basellaceae; es nativa de la región de los Andes (2800 - $4000 \mathrm{msnm}$ ) y ocurre desde Venezuela hasta Chile y Argentina. U. tuberosus es la única especie en el género Ullucus y son aceptados dos subespecies tuberosus, todas aquellas especies cultivables, y la subespecie aborigineus, que engloba a las especies silvestres ${ }^{2,3}$. Algunos autores afirman que es una planta domesticada hace 5500 años en la región de los $\mathrm{Andes}^{4}$ mientras que otros autores afirman que las poblaciones andinas la consumían por los $9000-8500$ años $\mathrm{AC}^{3}$. Es conocida bajo los nombres de papa lisa o chugua (Colombia), melloco, rubas (Ecuador), olluco, olloco, ullush, ulluco, ulluma, papa lisa, lisas e ilaco (Perú y Bolivia). U. tuberosus, dependiendo del país, además de ser una fuente alimenticia en la región de los Andes, también es usada en medicina tradicional para el tratamiento de diabetes, así como antiinflamatorio, analgésico y cicatrizante ${ }^{2}$. En el Perú la especie es usada en la alimentación y existen en diferentes colores que van desde el blanco, amarillo, anaranjado, rojo, morado y una variedad con manchas de diversos colores ${ }^{5}$.

Estudios de los metabolitos secundarios presentes en $U$. tuberosus han reportado la presencia de betalaínas ${ }^{6,5,7}$ y de las saponinas triterpénicas tuberosidas $\mathrm{A}, \mathrm{B}, \mathrm{y} \mathrm{C}^{2}$. El extracto bruto cetónico de la pulpa de los tubérculos presenta una excelente actividad cicatrizante ${ }^{4}$.

El presente trabajo reporta la presencia de los ésteres metílicos y los esteroides mayoritarios presentes en la fracción apolar del zumo liofilizado de $U$. tuberosus. La identificación de estos compuestos se realizó por análisis por cromatografía gaseosa acoplada a espectrómetro de masas (CG-EM) y por análisis de sus espectros de resonancia magnética nuclear de hidrógeno y de carbono 13 ( $\mathrm{RMN}$ de ${ }^{1} \mathrm{H}$ y $\mathrm{RMN}$ de ${ }^{13} \mathrm{C}$ ).

\section{PARTE EXPERIMENTAL}

\section{Muestra}

Los tubérculos de color anaranjado fueron comprados de un productor de la región del Callejón de Huaylas, en los alrededores de Huaraz. En la tabla 1 presentamos la clasificación taxonómica de $U$. tuberosus y en la figura 1 presentamos la foto de la especie en estudio. 
Tabla 1. Taxonomía de Ullucus tuberosus Caldas

\begin{tabular}{ll}
\hline Reino & Plantae \\
División & Magnoliophyta \\
Clase & Magnoliopsida \\
Orden & Caryophyllales \\
Familia & Basellaceae \\
Género & Ullucus \\
Especie & Ullucus tuberosus Caldas \\
\hline
\end{tabular}

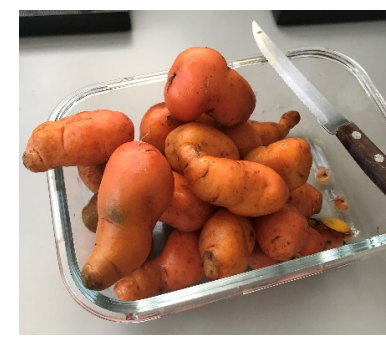

Figura 1. Foto de los tubérculos de Ullucus tuberosus Caldas.

\section{Extracción e identificación de los metabolitos secundarios}

Los tubérculos fresco de $U$. tuberosus fueron higienizados, secados y pesados (4 kg). A través de un extractor fue obtenido el zumo y el bagazo, los que fueron congelados y liofilizados.

El zumo liofilizado $(285,14 \mathrm{~g})$ fue macerado con metanol $(\mathrm{MeOH})$ tres veces. El metanol del extracto orgánico se evaporó en campana, obteniéndose el extracto bruto orgánico (EBO) $(59,99 \mathrm{~g})$; el EBO fue sometido a partición líquido-líquido, a partir del cual se obtuvieron los residuos, clorofórmico $\left(\mathrm{RCHCl}_{3}\right)$ 1,2 g, butanólico $(\mathrm{RBuOH}) 12,9 \mathrm{~g}$ y acuoso $\left(\mathrm{RH}_{2} \mathrm{O}\right)$ 45,89 g. Del $\mathrm{RCHCl}_{3}$ se tomó $278,7 \mathrm{mg}$ para ser sometido a fraccionamiento en cromatografía en columna usando como fase estacionaria sílica-gel fase normal y como fase móvil se usó éter de petróleo $(\mathrm{EP})$, cloroformo $\left(\mathrm{CHCl}_{3}\right)$ y $\mathrm{MeOH}$, obteniéndose así, la fracción de éter de petróleo $(\mathrm{FEP})$, la fracción clorofórmica $(\mathrm{FCHCl} 3)$ y la fracción metanólica $(\mathrm{FMeOH})$.

Para la identificación de los ácidos grasos presentes en los tubérculos fue necesario hacer una transesterificación de la FEP. La transesterificación fue siguiendo las normas del Instituto Adolf Lutz ${ }^{8}$. A aproximadamente $25 \mathrm{mg}$ de FEP se adicionó $3 \mathrm{~mL}$ de $\mathrm{n}$-hexano y $15 \mathrm{~mL}$ de la solución de ácido sulfúrico al $2 \%$ en metanol. La mezcla fue sometida a reflujo por una hora, luego fue enfriada y adicionada sobre solución saturada de cloruro de sodio hasta que la fase hexánica llegue hasta el nivel pre-establecido en una fiola. Los ésteres metílicos formados se encuentran en la fase hexánica, que fue concentrada y analizada por CG-EM 9 . 
La masa restante del $\mathrm{RCHCl}_{3}(918,8 \mathrm{mg})$ fue sometida a fraccionamiento usando como fase estacionaria sephadex LH-20 y como fase móvil fue EP, diclorometano (DCM) y $\mathrm{MeOH}$, obteniéndose las fracciones de fracción de éter de petróleo (FEP-1 con masa de 319,49 mg), fracción de diclorometano (FDCM con masa de 86,7 mg) y fracción de metanol (FMeOH-1 con masa de 512,61 mg).

La FEP-1 fue sometida a fraccionamiento, usando como fase estacionaria sílica-gel fase normal y como fase móvil la mezcla de los solventes $\mathrm{EP}$, acetato de etilo (AcOEt), $\mathrm{CHCl}_{3}$ y $\mathrm{MeOH}$ de polaridad creciente, se obtuvieron 64 fracciones, que fueron sometidas a cromatografía en capa delgada analítica en cromatofolios de sílica-gel y revelando con una solución de ácido sulfúrico al $20 \%$, seguida de calentamiento. Luego fueron reunidas siguiendo un patrón de semejanza, obteniéndose las fracciones 27 - 29 y 35 - 36, ambas fracciones fueron sometidas a análisis por resonancia magnética nuclear para su identificación.

\section{Análisis por cromatografía gaseosa acoplada a espectrómetro de masas}

Para los ésteres metílicos.- El análisis por cromatografía gaseosa fue realizado en un cromatógrafo a gas acoplado a un espectrómetro de masas (Shimadzu, modelo CGMS-QP2010 Ultra) con las siguientes condiciones de análisis: columna capilar Restek Rtx-5MS (30 m x 0,25 $\mathrm{mm} \times 0,25 \mu \mathrm{m}$ ); temperatura del inyector $240^{\circ} \mathrm{C}$, temperatura del detector $230{ }^{\circ} \mathrm{C}$; impacto de electrones a $70 \mathrm{eV}$, con gas de arrastre helio a un flujo de $1,23 \mathrm{~mL} / \mathrm{min}$, con split $1 / 5$; con un programa de temperatura de $120^{\circ} \mathrm{C}\left(2^{\prime}\right)-280^{\circ} \mathrm{C}, 2^{\circ} \mathrm{C} /$ minuto; y con volumen de inyección de $3 \mu \mathrm{L}$.

La identificación de las sustancias componentes fue a través de la comparación de los espectros de masas con el banco de datos del sistema de CG-EM de NIST 62 lib. y con la inyección de los siguientes patrones: hidrocarbonos (Mixture for GC, 98 \%, Lot: 65H5950, Sigma-Aldrich).

\section{Análisis por resonancia magnética nuclear}

Los espectros de resonancia magnética nuclear de ${ }^{1} \mathrm{H}$ y de ${ }^{13} \mathrm{C}$ fueron registrados en un espectrómetro Bruker de $500 \mathrm{MHz}$, operando a $500 \mathrm{MHz}$ para los espectros de hidrógeno y para la obtención de los espectros de carbono 13 operaba a $125 \mathrm{MHz}$. Los espectros fueron obtenidos en cloroformo deuterado $\left(\mathrm{CDCl}_{3}\right)$ de la marca MERCK.

\section{RESULTADOS Y DISCUSIÓN}

El zumo obtenido del material fresco fue liofilizado, demostrando que el 90,2 \% es agua y el material obtenido después de la liofilización fue un sólido amarillo $(285,14 \mathrm{~g})$, que al ser macerado con $\mathrm{MeOH}$ se obtuvo un extracto de color amarillo intenso, la maceración se realizó tres veces consecutivas. La tercera maceración fue levemente amarilla. El EBO obtenido fue de 59,99 g $(1,49 \%)$ de color amarillo intenso y de olor característico del $U$. tuberosus dulce. 
La FEP del $\mathrm{RCHCl}_{3}$, después de ser sometida a transesterificación para obtener los ésteres metílicos presentes en el zumo de U. tuberosus, fue analizada por CG-EM, obteniéndose un pico intenso (con tiempo de retención 31,656 min) en el cromatograma de iones totales (figura 2) que representa el 83,91 \% de la FEP y fue identificado como el éster metílico del ácido palmítico por análisis de su espectro de masas.

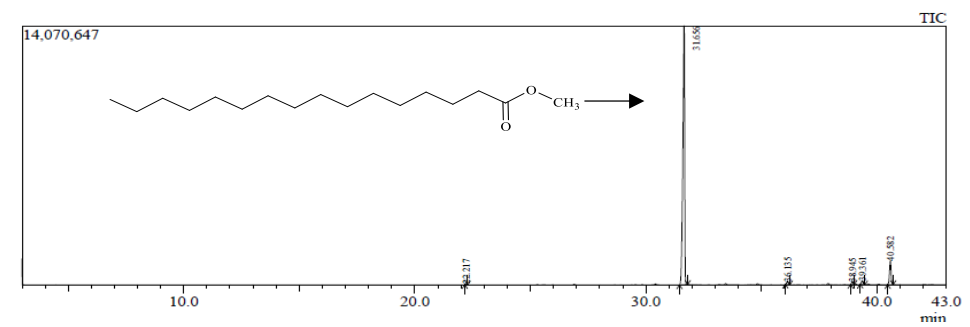

Figura 2. Cromatograma de iones totales obtenido por CG-EM de los ésteres metílicos del zumo de los tubérculos de U. tuberosus.

Con el análisis de los espectros de $\mathrm{RMN}-{ }^{1} \mathrm{H}$ y de $\mathrm{RMN}-{ }^{13} \mathrm{C}$ de las fracciones $27-29$ y 35 - 36 fueron identificados los esteroides estigmasterol (1) para la primera fracción y espinasterol (2) para la segunda fracción.

El espinasterol ya fue aislado de otras plantas como espinacas, Samanea saman y este esteroides presenta actividad antiproliferativa de las células $\mathrm{CACO}-2$, con una concentración mínima inhibitoria de $60 \mathrm{nM} / \mathrm{mL}$, también tiene un significativo potencial terapéutico para modular el desarrollo y/o progreso de la nefropatía diabética, antiinflamatorio, antitumoral, antioxidante, antiulcerogénico ${ }^{10}$.

Compuesto 1.- Se obtuvo 9,3 mg de unos cristales transparentes. Por análisis de sus desplazamientos químicos de sus espectros de RMN se llegó a la conclusión de que la muestra es el esteroide estigmasterol (figura 3). $\mathrm{RMN}^{-1} \mathrm{H}: \delta 0,68(3 \mathrm{H}, \mathrm{s}, \mathrm{H}-18), 0,80(3 \mathrm{H}, \mathrm{d}$, $J=6,75 \mathrm{~Hz}, \mathrm{H}-27), 0,81$ (3H, t, $J=7,5 \mathrm{~Hz}, \mathrm{H}-29), 0,85$ (3H, d, $J=6,7 \mathrm{~Hz} \mathrm{H}-26), 1,00$ (3H, s, H-19), 1,01 (3H, d, $J=6,7 \mathrm{~Hz}, \mathrm{H}-21), 3,59(1 \mathrm{H}, \mathrm{m}, \mathrm{H}-3), 5,01(1 \mathrm{H}, \mathrm{dd}, J=14,95,8,5 \mathrm{~Hz}$, $\mathrm{H}-22), 5,15(1 \mathrm{H}, \mathrm{dd}, J=15,25,8,85 \mathrm{~Hz}, \mathrm{H}-23), 5,35(1 \mathrm{H}, \mathrm{m}, \mathrm{H}-6) . \mathrm{RMN}-{ }^{13} \mathrm{C}: \delta 21,21(\mathrm{C}-$ 18), 12,31 (C-29), 18,97 (C-27), 19,38 (C-19), 21,06 (C-11, C-26), 21,25 (C-21, C-27), 24,28 (C-15), 25,38 (C-28), 28,91 (C-16), 31,52 (C-2), 31,87 (C-7), 32, 07 (C-8, C-25), 36,51 (C10), 37,01 (C-1), 39,95 (C-12), 40,49 (C-20), 42,29 (C-13), 42,49 (C-4), 49,52 (C-9), 51,23 (C-24), 56,1 (C-17), 56,61 (C-14),71,39 (C-3), 121, 72 (C-6), 129,22 (C-23), 138,35 (C-22) y $140,75(\mathrm{C}-5)^{11}$. 


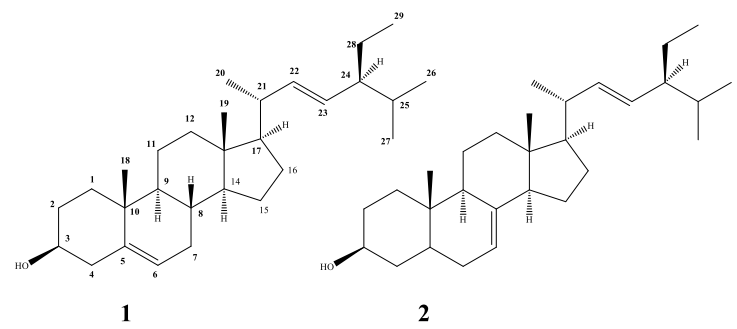

Figura 3. Esteroides identificados en el zumo de los tubérculos de U. tuberosus.

Compuesto 2.- Se obtuvo 6,2 mg de un sólido blanco, por análisis de sus espectros de RMN se llegó a la conclusión que la muestra fue identificada como espinasterol (figura 3). RMN- ${ }^{1} \mathrm{H}: \delta$ 0,56 (3H, s, Me-18), 0,80 (3H, s, Me-19), 0,81 (3H, d, J = 6,5 Hz, H-27), 0,82 (3H, t, J= 7,3 $\mathrm{Hz}, \mathrm{H}-29), 0,86$ (3H, d, $J=6,7 \mathrm{~Hz}, \mathrm{Me}-26), 1,04$ (3H, d, $J=6,4 \mathrm{~Hz}, \mathrm{Me}-21), 3,60(1 \mathrm{H}, \mathrm{m}$, H-3), 5,03 (1H, dd, $J=15,25 ; 8,55, \mathrm{H}-23), 5,16(1 \mathrm{H}, d d, J=15,25 ; 8,85, \mathrm{H}-22), 5,17$ (1H, m, H-7). RMN-13C: $\delta$ 12,04 (C-18), 12,23 (C-29), 13,03 (C-19), 18,98 (C-27), 21,07 (C-26), 21,36 (C-21), 21,55 (C-11), 23,01 (C-15), 25,39 (C-28), 28,48 (C-16), 29,65 (C-6), 31,48 (C2), 31,87 (C-25), 34,23 (C-10), 37,15 (C-1), 38,01 (C-4), 39,47 (C-12), 40,26 (C-5), 40,79 (C-20), 43,28 (C-13), 49,46 (C-9), 51,25 (C-24), 55,13 (C-14), 55,85 (C-17), 71,06 (C-3), 117,46 (C-7), 129,46 (C-23), 138,15 (C-22) y 139,57 (C-8) los desplazamientos químicos están conforme la referencia ${ }^{11}$.

\section{CONCLUSIONES}

El estudio fitoquímico del zumo de los tubérculos de U. tuberosus de las fracciones apolares permitió la identificación del ácido graso palmítico como el mayoritario. También fueron identificados los esteroides estigmasterol y espinasterol. Es la primera vez que se reporta la presencia de estas moléculas en los tubérculos de la especie en estudio.

\section{AGRADECIMIENTOS}

Los autores agradecen al Vicerrectorado de Investigación de la Universidad Nacional de Ingeniería, por el apoyo económico brindado para la realización de este trabajo de investigación (Proyecto de Investigación Formativa 2018 código: FIQT-F-1-2018). 


\section{REFERENCIAS BIBLIOGRÁFICAS}

1. Suquilanda M. Producción orgánica de cultivos andinos. Quito, Ecuador: Organización de las Naciones Unidas para la Alimentación y la Agricultura; 2012.

2. Espada A, Jiménez C, Dopeso J, Riguera R. Tuberosides A, B, and C, Novel triterpenoid saponins from the hypoglucaemic fraction of Ullucus tuberosus. Liebigs Annalen. 1996; 5: 781-784. https://doi.org/10.1002/jlac.199619960523

3. Manrique I, Arbuzi C, Vivanco F, Gonzales R, Chávez O, Tay D, et al. Colección de germoplasma de Ullucu conservada en el Centro Internacional de la Papa (CIP). Lima, Perú: Centro Internacional de la Papa (CIP); 2017.

4. Heil N, Bravo K, Montoya A, Robledo S, Osorio E. Wound healing activity of Ullucus tuberosus, an Andean tuber crop. Asian Pac J Trop Biomed. 2017; 7(6): 538-543.

5. Sveson J, Smallfield BM, Joyce NI, Sansom CE, Perry NI. Betalains in red and yellow varieties of the Andean tuber crop Ulluco (Ullucus tuberosus). J Agr Food Chem. 2008; 56: 7730-7737. https://doi.org/10.1021/jf8012053

6. Cejudo-Bastante MJ, Hurtado N, Mosquera N, Heredia FJ. Potential use of new Colombian sources of betalains. Color stability of ulluco (Ullucus tuberosus) extracts under different $\mathrm{pH}$ and thermal conditions. Food Res Int. 2014; 64: 465-471.

7. Campos D, Noratto G, Chirinos R, Arbizu C, Roca W, Cisneros-Zevallos L. Antioxidant capacity and secondary metabolites in four species of Andean tuber crops: native potato (Solanum sp.), mashua (Tropaeolum tuberosum Ruiz \& Pavón), Oca (Oxalis tuberosa Molina) and ulluco (Ullucus tuberosus Caldas). J Sci Food Agric. 2006; 86: 1481-1488.

8. Instituto Adolf Lutz. Normas analíticas. 3 Edição. São Paulo: IMESP; 1985. p 245-266

9. Díaz IEC, Gonçalves EG, Marques MOM, Yoshida M. Constituyentes químicos del túbero de Dracontium spruceanum (Schott) G. Zhu ex Dracontium loretense Krause (Araceae). Rev Soc Quím Perú. 2011; 77(4): 275-284.

10. Ragasa CY, Galian RF, Arenal M, Tan V, Shen Ch-Ch. Chemical constituents of Petersianthus quadrialatus (Merr.). Res J Pharm Biol Chem Sci. 2014; 5(4): 1501 1507.

11. Kojima H, Sato N, Hatano A, Ogura H. Sterol glucosides from Prunella vulgaris Phytochem. 1990; 29(7): 2351-2355. 